\title{
Survival of patients with multidrug-resistant TB in Eastern Europe: what makes a difference?
}

\author{
Yanina Balabanova, ${ }^{1,2,3}$ Olga Ignatyeva, ${ }^{4}$ Lena Fiebig, ${ }^{3}$ Vija Riekstina, ${ }^{5}$ \\ Manfred Danilovits, ${ }^{6}$ Kadri Jaama, ${ }^{6}$ Edita Davidaviciene, ${ }^{7}$ Birute Radiulyte, ${ }^{7}$ \\ Christina Marcela Popa, ${ }^{8}$ Vladyslav Nikolayevskyy, ${ }^{1,2}$ Francis Drobniewski ${ }^{1,2}$
}

\begin{abstract}
- Additional material is published online only. To view please visit the journal online (http://dx.doi.org/10.1136/ thoraxjnl-2015-207638).

For numbered affiliations see end of article.
\end{abstract}

\section{Correspondence to}

Yanina Balabanova, Department for Infectious Disease Epidemiology, Robert Koch Institute, Berlin 13353, Germany; LenzY@rki.de

Received 29 July 2015 Revised 26 February 2016 Accepted 28 February 2016 Published Online First 24 March 2016
CrossMark

\footnotetext{
To cite: Balabanova $Y$, Ignatyeva 0 , Fiebig $L$, et al. Thorax 2016;71:854-861.
}

\begin{abstract}
Background The quality of care for patients with TB in Eastern Europe has improved significantly; nevertheless drug resistance rates remain high. We analysed survival in a cohort of patients with multidrug-resistant and extensively drug-resistant (MDR-/XDR-) TB from Latvia, Lithuania, Estonia and Bucharest city.

Methods Consecutive adult new and retreatment patients with culture-confirmed pulmonary MDR-TB registered for treatment in 2009 (and in 2007 in Latvia) were enrolled; prospective survival information was collected.
\end{abstract}

Results A total of 737 patients were included into the cohort. Of all MDR-TB cases, 46\% were newly diagnosed; $56 \%$ of all MDR-TB cases had no additional resistance to fluoroquinolones or injectable agents, 33\% had pre-XDR-TB and $11 \%$ XDR-TB. Median survival was 5.9 years in patients with MDR-TB and XDR-TB; 1.9 years in patients coinfected with HIV. Older age, male gender, alcohol abuse, retirement, co-morbidities, extrapulmonary involvement and HIV coinfection independently worsened survival. Inclusion of fluoroquinolones and injectable agents improves survival in patients with MDR-TB. Pre-XDR and XDR status did not significantly shorten survival as long as fluoroquinolones and injectable agents were part of the regimen. Moxifloxacin seems to improve survival in ofloxacin-susceptible patients when compared with older generation fluoroquinolones.

Conclusions The burden of additional resistances in patients with MDR-TB is high likely due to primary transmission of resistant strains. Social and programmatic factors including management of alcohol dependency, expansion of HIV testing and antiretroviral treatment need to be addressed in order to achieve cure and to interrupt transmission. The role of last generation fluoroquinolones and injectable agents in treatment of patients with pre-XDR and XDR-TB needs to be further investigated.

\section{INTRODUCTION}

TB remains an international public health emergency with 1.5 million deaths annually. Although global progress has been made towards the 2015 Millennium Development Goals with steadily decreasing TB incidence, prevalence and mortality rates, the rates of $\mathrm{TB}$ resistant to at least isoniazid (INH) and rifampicin (RIF) (multidrug-resistant TB, MDR-TB) including cases with further

\section{Key messages}

What is the key question?

- How long is the survival in a cohort of patients infected with multidrug-resistant and extensively drug-resistant (MDR-/XDR-)TB in Latvia, Lithuania Estonia and Romania and what are the factors influencing survival including resistance pattern and treatment regimen?

\section{What is the bottom line?}

- Survival of patients with MDR-TB is short and is little influenced by additional drug resistance; however inclusion of fluoroquinolones in particular of a later generation and injectable agents improves survival.

\section{Why read on?}

- This recent multicentre study provides an update on epidemiological aspects of the TB epidemic in Eastern Europe with implications for management of patients with MDR-TB/ XDR-TB globally.

resistance to a fluoroquinolone (FQ) and a secondline injectable drug (INJ) (extensively drug-resistant TB, XDR-TB) are growing being the world's highest in Eastern Europe. ${ }^{12}$

Aiming to avert the further development and transmission of drug resistance, the Baltic countries have been systematically tackling the problem of improving the quality of care for patients with TB. ${ }^{13}$ Latvia and Estonia have repeatedly reported high rates of treatment success for patients with MDR-TB and XDR-TB. ${ }^{2}{ }^{4-6}$ Nevertheless, the levels of drug resistance remain high $(8.8 \%$ among new cases and $26.0 \%$ among retreatment cases in Latvia, $11.0 \%$ and $44.0 \%$ in Lithuania, $17.0 \%$ and $48.0 \%$ in Estonia in 2014, respectively) significantly limiting the treatment options. The proportion of XDR among MDR-TB cases reaches 7.3\% in Estonia, $16.0 \%$ in Latvia and $24.8 \%$ in Lithuania. In Romania, the southeastern European country, the levels of drug-resistant TB, although being lower than in the Baltics; however still account for $2.8 \%$ of new and $11.0 \%$ of retreatment cases $;^{2}$ the TB mortality rates are many times 
higher than the average TB mortality rates in the European Union (EU). ${ }^{7}$ Substantial levels of additional drug resistance complicate the problem of MDR-TB management even further. ${ }^{1}{ }^{8}$ It is known that the molecular epidemiology of strains driving the TB epidemic in Eastern Europe is changing being increasingly driven by two homogenous clades of Beijing family of strains. ${ }^{9} 10$ The fast evolving epidemic of HIV adds an extra burden. ${ }^{11}$

Achieving successful treatment outcome in patients with MDR-TB and in particular XDR-TB remains a challenge and death is common. ${ }^{12}{ }^{13}$ Several studies looked at the factors associated with mortality in cohorts of patients with drugsusceptible and drug-resistant TB in the past. ${ }^{514-19}$ As the management strategies improve against the background of changing molecular epidemiology, a detailed analysis of recent cohorts looking at the survival of patients and factors influencing the length of survival is needed.

The present study was conducted within the framework of the EU-funded project 'TB-PAN-NET' and involved the Baltic countries and the city of Bucharest (Romania); its purpose was to establish an Eastern European MDR-TB/XDR-TB cohort and to determine long-term survival among it.

\section{METHODS}

\section{Study population}

This prospective 3-year study was conducted across four Eastern European settings: National Tuberculosis and Infectious Diseases University Hospital in Vilnius, Clinic of Tuberculosis and Lung Diseases at Riga East University hospital, Lung Hospital at Tartu University, Estonia and Marius Nasta Institute of Pneumology, Bucharest, Romania. Consecutive adult new and retreatment patients with MDR-TB and XDR-TB were enrolled. The sample included patients registered for treatment at each centre in 2009; in Latvia an additional 2007 cohort of patients with MDR-TB/XDR-TB was recruited. Demographic and clinical data were collected from each patient at the point each was registered for treatment within the recruitment years. Information was drawn from the national registry.

The patients were followed-up until 2012; long-term retrospective information was available for retreatment cases. The median follow-up time since first diagnosis of TB was 2.1 years in Lithuania, 2.8 years in Latvia, 2.3 years in Estonia and 2.4 years in Romania.

All specimens were tested locally (for Mycobacterium tuberculosis culture and drug-susceptibility testing (DST)); the initial DST is routinely performed on the first positive mycobacterial culture for all cases; a follow-up test is repeated if treatment failure or newly developed drug resistance is suspected on a specimen collected at least 30 days after the initial specimen. DST for first-line and second-line drugs (FLD; SLD) is performed using solid or automated liquid culture media system (BACTEC MGIT 960, Becton Dickinson, Sparks, Maryland, USA) according to standard procedures. ${ }^{20}$ All laboratories underwent annual quality assurance through the WHO Global Project on Drug Resistance and/or European Center for Disease Control European Reference Laboratory Network External Quality Assurance programme with good results.

New patients with MDR-TB were treated with a standardised regimen until DST results became known at which point patients were treated with a regimen based on in vitro drug susceptibility. Retreatment patients were treated initially with a drug regimen based on their old DST results and treatment regimens were modified when in vitro DST results became available.

\section{Data collection and management}

Structured questionnaires were used for anonymised collection of demographic and clinical data that were later double-entered into a password-protected Microsoft Access 2007 database on-site and rechecked by a coordinating team centrally.

\section{Statistical analysis}

We described categorical variables using numbers and percentages. Death was defined as death from any cause during treatment or follow-up from the time of the first-recorded diagnosis of MDR-TB/XDR-TB in the national TB registers until the censoring date in December 2012, the end of the investigation period. The date of the last visit to a TB clinic was recorded as the last day when a patient was documented to be alive.

We calculated mortality rates (per 100 person-years (PY)) and 95\% CIs and used Kaplan-Meier survival function to estimate survival probabilities. Crude and adjusted Cox proportional hazard regression was used to estimate the effect on survival of independent variables including treatment regimen, drug resistance pattern, sociodemographic and clinical characteristics of the patients assessed at the diagnosis. We looked at the effects of individual drugs and treatment combinations stratified by resistance pattern of the strains. The multivariable model for calculating adjusted HRs (aHRs) contained factors significant on univariable analysis ( $\mathrm{p}<0.05$, two-sided) with enough observations (across all sites).

All analyses were performed using Stata V.13 (Stata Corporation, College Station, Texas, USA).

\section{Ethics review}

The project was reviewed and approved by the Ethics Review Committees of the University of Tartu and Riga Stradins University and received a waiver of informed consent as anonymised data were used. Local Ethics Committees exempted the study from an ethics review in Lithuania and Romania. The study was approved by Queen Mary College Research Ethics Committee.

\section{RESULTS}

\section{Sociodemographic and clinical characteristics}

Survival information was available for $737 / 745$ recruited patients with MDR-TB (table 1).

Patients were broadly similar across the four study sites: the majority was urban males between 40 and 60 years of age. Only $25 \%$ of patients were employed. Most patients smoked, half of them abused alcohol. Of those tested for HIV $(n=530 ; 72 \%)$, $20(4 \%)$ were positive; the testing coverage was the lowest in Lithuania. More than half of the patients (62\%) had cavitary pulmonary TB. Only a few patients were tested for hepatitis B and $\mathrm{C}$ and of those 5/60 (8\%) and 14/67 (21\%) were positive, respectively.

Approximately half $(46 \%)$ of all patients with MDR-TB/ XDR-TB were new cases; however, in Estonia the majority (85\%) of the cases were newly diagnosed. Of those patients on retreatment the majority had between two and four treatment episodes and $54 \%$ of them had an unsuccessful treatment outcome in the past. In $71 \%$ of all patients with MDR-TB/XDR-TB culture conversion was achieved within the first 6 months of treatment; a further $15 \%$ converted within 12 months; $1 \%$ after 12 months and in $12 \%$ of patients culture conversion was not achieved. In $8 \%$ of cases surgery was performed. Of the 137 patients with data on treatment compliance only $43 \%$ were fully adherent throughout the treatment and the rest interrupted their treatment 
Table 1 Sociodemographic and clinical characteristic of multidrug-resistant and extensively drug-resistant TB cases across all study sites and mortality rates $(n=737)$

\begin{tabular}{|c|c|c|c|c|c|c|}
\hline & \multicolumn{3}{|c|}{ Total } & \multicolumn{3}{|l|}{ Mortality } \\
\hline & $\mathrm{n}$ & $\mathrm{N}$ & $\%$ & Person-years & Deaths & Rate; $95 \% \mathrm{Cl}$ \\
\hline \multicolumn{7}{|l|}{ Demographic factors } \\
\hline \multicolumn{7}{|l|}{ Age group (years) } \\
\hline $15-29$ & 109 & 737 & 15 & 294 & 13 & 4.42 (2.57 to 7.62$)$ \\
\hline $30-39$ & 148 & 737 & 20 & 451 & 37 & 8.20 (5.94 to 11.31$)$ \\
\hline $40-49$ & 192 & 737 & 26 & 532 & 62 & 11.66 (9.09 to 14.96$)$ \\
\hline $50-59$ & 196 & 737 & 27 & 576 & 72 & $12.50(9.92$ to 15.75$)$ \\
\hline$\geq 60$ & 92 & 737 & 12 & 221 & 43 & 19.46 (14.43 to 26.24$)$ \\
\hline \multicolumn{7}{|l|}{ Gender } \\
\hline Male & 581 & 737 & 79 & 1575 & 195 & 12.38 (10.76 to 14.25$)$ \\
\hline Female & 156 & 737 & 21 & 499 & 32 & 6.41 (4.53 to 9.06$)$ \\
\hline \multicolumn{7}{|l|}{ Living settings } \\
\hline Urban & 499 & 737 & 68 & 1405 & 149 & 10.61 (9.04 to 12.46$)$ \\
\hline Rural & 238 & 737 & 32 & 670 & 78 & 11.65 (9.33 to 14.54$)$ \\
\hline \multicolumn{7}{|l|}{ Social factors } \\
\hline \multicolumn{7}{|l|}{ Employment } \\
\hline Unemployed & 481 & 736 & 65 & 1394 & 173 & 12.41 (10.69 to 14.4$)$ \\
\hline Retired & 71 & 736 & 10 & 182 & 31 & 17.00 (11.96 to 24.17$)$ \\
\hline Employed & 184 & 736 & 25 & 495 & 23 & 4.65 (3.09 to 6.99$)$ \\
\hline \multicolumn{7}{|l|}{ Homelessness } \\
\hline Homeless & 54 & 737 & 7 & 144 & 17 & 11.79 (7.33 to 18.97$)$ \\
\hline Have home & 683 & 737 & 93 & 1930 & 210 & 10.88 (9.5 to 12.46$)$ \\
\hline \multicolumn{7}{|l|}{ Substance abuse* } \\
\hline \multicolumn{7}{|l|}{ Smoking } \\
\hline Yes & 434 & 570 & 76 & 1229 & 152 & 12.37 (10.55 to 14.5$)$ \\
\hline No & 136 & 570 & 24 & 363 & 27 & 7.44 (5.1 to 10.85$)$ \\
\hline \multicolumn{7}{|l|}{ Alcohol abuse } \\
\hline Yes & 421 & 731 & 58 & 1182 & 170 & 14.38 (12.37 to 16.71$)$ \\
\hline No & 310 & 731 & 42 & 868 & 53 & 6.10 (4.66 to 7.99$)$ \\
\hline \multicolumn{7}{|l|}{ Recreational drugs } \\
\hline Yes & 37 & 719 & 5 & 98 & 11 & 11.23 (6.22 to 20.29 ) \\
\hline No & 682 & 719 & 95 & 1928 & 208 & $10.79(9.42$ to 12.36$)$ \\
\hline \multicolumn{7}{|l|}{ Co-morbiditiest } \\
\hline \multicolumn{7}{|l|}{ HIV } \\
\hline Negative & 510 & 737 & 69 & 1463 & 141 & 9.64 (8.17 to 11.37 ) \\
\hline Positive & 20 & 737 & 3 & 33 & 10 & 30.51 (16.41 to 56.7$) \ddagger$ \\
\hline Not tested & 207 & 737 & 28 & 579 & 76 & 13.14 (10.49 to 16.45$)$ \\
\hline \multicolumn{7}{|l|}{ Condition other than HIV $†$} \\
\hline Yes & 37 & 737 & 5 & 81 & 22 & 27.10 (17.84 to 41.16$) \ddagger$ \\
\hline No & 700 & 737 & 95 & 1993 & 205 & 10.29 (8.97 to 11.8$)$ \\
\hline \multicolumn{7}{|l|}{ Disease characteristics } \\
\hline \multicolumn{7}{|l|}{ New or retreatment case } \\
\hline New case & 336 & 737 & 46 & 732 & 79 & 10.79 (8.65 to 13.45$)$ \\
\hline Retreatment case & 401 & 737 & 54 & 1342 & 148 & 11.03 (9.39 to 12.96$)$ \\
\hline Pulmonary or extrapulmonary & & & & & & \\
\hline Pulmonary only & 705 & 737 & 96 & 2010 & 212 & 10.55 (9.22 to 12.07$)$ \\
\hline Both pulmonary and extrapulmonary & 32 & 737 & 4 & 64 & 15 & 23.47 (14.15 to 38.94$) \ddagger$ \\
\hline Drug resistance pattern§ & & & & & & \\
\hline MDR & 395 & 705 & 56 & 1031 & 113 & 10.96 (9.12 to 13.18$)$ \\
\hline MDR+FQres & 43 & 705 & 6 & 149 & 15 & 10.06 (6.07 to 16.69$)$ \\
\hline MDR+INJres & 186 & 705 & 26 & 510 & 52 & $10.19(7.77$ to 13.37$)$ \\
\hline $\mathrm{XDR}$ & 81 & 705 & 11 & 307 & 36 & $11.73(8.46$ to 16.26$)$ \\
\hline Presence of cavities & & & & & & \\
\hline Yes & 456 & 732 & 62 & 1278 & 149 & 11.66 (9.93 to 13.69$)$ \\
\hline No & 276 & 732 & 38 & 785 & 77 & 9.81 (7.85 to 12.27$)$ \\
\hline
\end{tabular}


Table 1 Continued

\begin{tabular}{|c|c|c|c|c|c|c|}
\hline & \multicolumn{3}{|c|}{ Total } & \multicolumn{3}{|l|}{ Mortality } \\
\hline & $\mathbf{n}$ & $\mathrm{N}$ & $\%$ & Person-years & Deaths & Rate; $95 \% \mathrm{Cl}$ \\
\hline \multicolumn{7}{|c|}{ Smear positivity at diagnosis } \\
\hline Yes & 573 & 737 & 78 & 380 & 28 & 7.37 (5.09 to 10.67$)$ \\
\hline No & 164 & 737 & 22 & 337 & 31 & 9.19 (6.46 to 13.06$)$ \\
\hline \multicolumn{7}{|c|}{ Unsuccessful treatment outcome in the past } \\
\hline Yes & 216 & 401 & 54 & 885 & 92 & 10.40 (8.48 to 12.75$)$ \\
\hline No & 185 & 401 & 46 & 457 & 56 & $12.26(9.43$ to 15.93$)$ \\
\hline
\end{tabular}

for a period between 1 day and 2 months but not qualifying to be considered as lost to follow-up.

Fifty-six per cent of cases had MDR-TB without any resistance to FQs or INJs; however, of these cases 95\% had additional drug resistance to other anti-TB drugs, so that only 5\% of cases were MDR-TB with resistance to only INH and RIF. Of the remaining 44\% patients with MDR-TB, 33\% had pre-XDR-TB (6\% resistant to FQ and $27 \%$ resistant to INJ only); 11\% had XDR-TB (see online supplementary table S1 with per-site patients' characteristics). Among 661 patients with data on treatment and drug resistance, the median number of drugs received was 5 (IQR 4-6). The majority of patients (503/ $661 ; 76 \%)$ received an INJ; 93\% (616/661) received FQs but in only $18 \%(109 / 616)$ this was a later generation FQ: moxifloxacin. The most commonly administered drug combinations were INJ and FQ combined with prothionamide/ethionamide (PT/ ETH), $p$-aminosalicylic acid (PAS) or cycloserine (CS), and possibly amoxicillin/clavulanate (73\%) and FQ with ETH/PT, PAS or CS and possibly amoxicillin/clavulanate (19\%). A negligible proportion of patients received clarithromycin $(18 ; 3 \%)$ or linezolid $(8 ; 1 \%)$ (table 2 ).

\section{Survival analysis}

The total follow-up period was 2074.1 PY. Across the whole sample 227 patients died giving an average mortality rate of 10.94 per 1000 patients with TB per year (95\% CI 9.61 to 12.47). The mortality rates were the highest among HIV-positive patients (30.51) and patients with other co-morbidities (27.10) including other immunocompromised conditions (29.24) and cancer (33.06) as well as those with extrapulmonary involvement (23.47) (table 1).

In unadjusted analyses, survival was shorter in men than women (see online supplementary figure S1A). The median survival of patients coinfected with HIV was 1.9 (95\% CI 0.3 to $\propto)$ years vs 5.9 (95\% CI 5.3 to 7.4 ) in HIV-negative patients (see online supplementary figure 1B) but was not markedly decreased in XDR-TB compared with patients with MDR-TB (5.9 (95\% CI 5.0 to 7.8 ) and 5.9 (95\% CI 4.7 to 6.9)) median years (see online supplementary figure $1 \mathrm{C}$ ).

The mortality rates are the highest when INJs and FQs are not part of the treatment being 58.56 across the whole sample, irrespective of the resistance pattern. In the patients with pre-XDR mortality rates are lower when an agent from a group to which an isolate is susceptible is added to the regimen; in all resistance groups including XDR, the regimen that includes both INJs and FQs dramatically lowers the mortality rates to
8.09 across the whole sample (see table 3 and online supplementary figure 1D).

In adjusted Cox regression analysis $(\mathrm{N}=657$, table 4$)$ older age (aHR >2 starting from 40 y.o. strata), male gender (aHR 2.00 (95\% CI 1.27 to 3.14)), alcohol abuse (aHR 1.70 (95\% CI 1.16 to 2.47$)$ ), retirement (aHR 2.69 (95\% CI 1.47 to 4.94$)$ ), presence of co-morbidities (aHR 2.33 95\% CI (1.34 to 4.05)) and extrapulmonary involvement (aHR 2.65 (95\% CI 1.45 to 4.83)) were independently associated with worse survival. HIV coinfection profoundly affected survival (aHR 2.53 (95\% CI 1.19 to 5.39 )). Smear positivity at diagnosis had no significant effect on survival (aHR 1.24 (95\% 0.83 to 1.86).

Independently from these factors, regimens that did not contain FQs or INJs were associated with a higher risk of dying in all resistance groups. Inclusion of INJs seems to improve survival in patients with MDR-TB with resistance to FQs including patients with XDR-TB. Inclusion of FQs in cases of INJ resistance as well as in cases of FQ resistance also seems to make a difference (although not reaching statistical significance).

However, when looking at the generation of FQ used, moxifloxacin was significantly associated with better survival in patients susceptible to ofloxacin $(\mathrm{N}=509)$ in vitro compared with those receiving older generation FQs univariably (HR 0.47 (95\% CI 0.23 to 0.97$)$ ); yet not significant when adjusted for all factors as above (aHR 0.54 (95\% CI 0.26 to 1.12)). For ofloxacin-resistant patients $(\mathrm{N}=105)$, administration of moxifloxacin did not improve survival when compared with other FQs (HR 1.00 (95\% CI 0.53 to 1.89 )).

The mortality rates and adjusted analyses show that the survival was similar in patients with MDR, pre-XDR as well as XDR-TB as long as INJs and FQs were included in the treatment indicating that the treatment has a bigger influence on survival than the resistance pattern.

\section{DISCUSSION}

Eastern European countries remain a hotspot for the drug-resistant TB within the EU. ${ }^{1}$ This multicentre study explores the survival and associated risk factors in a large representative cohort of patients with MDR-TB/XDR-TB from Latvia, Lithuania, Estonia and the city of Bucharest in light of improved diagnostics and patients' management, changing epidemiology of circulating strains and an increasing number of individuals coinfected with HIV.

The study results confirm a substantial burden of XDR-TB with ongoing active transmission of drug-resistant strains. The latter supports the hypothesis that mutations coding drug 
Table 2 Drug resistance profile and received treatment with second-line drugs according to the different drug resistance patterns

\begin{tabular}{|c|c|c|c|c|c|c|c|c|c|c|c|c|c|c|c|}
\hline \multirow[b]{3}{*}{ SLDs and combinations } & \multicolumn{15}{|c|}{ Drug resistance pattern } \\
\hline & \multicolumn{3}{|c|}{ MDR } & \multicolumn{3}{|c|}{ MDR+FQres } & \multicolumn{3}{|c|}{ MDR+INJres } & \multicolumn{3}{|c|}{ XDR } & \multicolumn{3}{|c|}{ Total } \\
\hline & $\mathbf{n}$ & $\mathrm{N}$ & Per cent & n & N & Per cent & n & $\mathrm{N}$ & Per cent & $\mathrm{n}$ & $\mathrm{N}$ & Per cent & $\mathbf{n}$ & N & Per cent \\
\hline \multicolumn{16}{|l|}{ Individual drugs } \\
\hline Injectables & 284 & 367 & 77 & 32 & 41 & 78 & 135 & 179 & 75 & 52 & 74 & 70 & 503 & 661 & 76 \\
\hline Amikacin & 24 & 367 & 7 & 10 & 41 & 24 & 11 & 179 & 6 & 10 & 74 & 14 & 55 & 661 & 8 \\
\hline Kanamycin & 231 & 367 & 63 & 18 & 41 & 44 & 48 & 179 & 27 & 18 & 74 & 24 & 315 & 661 & 48 \\
\hline Capreomycin & 85 & 367 & 23 & 16 & 41 & 39 & 97 & 179 & 54 & 28 & 74 & 38 & 226 & 661 & 34 \\
\hline Fluorochinolones & 335 & 367 & 91 & 37 & 41 & 90 & 176 & 179 & 98 & 68 & 74 & 92 & 616 & 661 & 93 \\
\hline Ofloxacin & 301 & 367 & 82 & 27 & 41 & 66 & 163 & 179 & 91 & 52 & 74 & 70 & 543 & 661 & 82 \\
\hline Moxifloxacin & 20 & 367 & 5 & 16 & 41 & 39 & 41 & 179 & 23 & 32 & 74 & 43 & 109 & 661 & 16 \\
\hline Levofloxacin & 4 & 367 & 1 & 0 & 41 & 0 & 4 & 179 & 2 & 1 & 74 & 1 & 9 & 661 & 1 \\
\hline Ciprofloxacin & 50 & 367 & 14 & 4 & 41 & 10 & 22 & 179 & 12 & 4 & 74 & 5 & 80 & 661 & 12 \\
\hline PT/ETH & 319 & 367 & 87 & 34 & 41 & 83 & 159 & 179 & 89 & 63 & 74 & 85 & 575 & 661 & 87 \\
\hline CS & 285 & 367 & 78 & 34 & 41 & 83 & 164 & 179 & 92 & 66 & 74 & 89 & 549 & 661 & 83 \\
\hline PAS & 174 & 367 & 47 & 28 & 41 & 68 & 136 & 179 & 76 & 55 & 74 & 74 & 393 & 661 & 59 \\
\hline Amoxicillin/clavulanate & 22 & 367 & 6 & 6 & 41 & 15 & 61 & 179 & 34 & 24 & 74 & 32 & 113 & 661 & 17 \\
\hline Linezolid & 1 & 367 & 0 & 2 & 41 & 5 & 0 & 179 & 0 & 5 & 74 & 7 & 8 & 661 & 1 \\
\hline Clarithromycin & 2 & 367 & 1 & 6 & 41 & 15 & 8 & 179 & 4 & 2 & 74 & 3 & 18 & 661 & 3 \\
\hline \multicolumn{16}{|l|}{ Drug combinations } \\
\hline Type 1: regimen does not include INJ or FQ & 24 & 367 & 7 & 0 & 41 & 0 & 1 & 179 & 1 & 3 & 74 & 4 & 28 & 661 & 4 \\
\hline Only FLDs & 22 & 367 & 6 & 0 & 41 & 0 & 1 & 179 & 1 & 0 & 74 & 0 & 23 & 661 & 3 \\
\hline Only PT/ETH and/or PAS and/or CS & 2 & 367 & 1 & 0 & 41 & 0 & 0 & 179 & 0 & 3 & 74 & 4 & 5 & 661 & 1 \\
\hline Type 2: regimen includes INJ & 9 & 367 & 2 & 4 & 41 & 10 & 2 & 179 & 1 & 3 & 74 & 4 & 18 & 661 & 3 \\
\hline INJ only & 1 & 367 & 0 & 0 & 41 & 0 & 0 & 179 & 0 & 0 & 74 & 0 & 1 & 661 & 0 \\
\hline INJ+(PT/ETH or PAS or CS) & 8 & 367 & 2 & 4 & 41 & 10 & 1 & 179 & 1 & 31 & 74 & 42 & 16 & 661 & 2 \\
\hline INJ+(PT/ETH or PAS or CS)+AMOX & 0 & 367 & 0 & 0 & 41 & 0 & 1 & 179 & 1 & 0 & 74 & 0 & 1 & 661 & 0 \\
\hline Type 3: regimen includes FQ & 59 & 367 & 16 & 9 & 41 & 22 & 43 & 179 & 24 & 19 & 74 & 26 & 130 & 661 & 20 \\
\hline FQ only & 1 & 367 & 0 & 1 & 41 & 2 & 0 & 179 & 0 & 0 & 74 & 0 & 2 & 661 & 0 \\
\hline $\mathrm{FQ}+(\mathrm{PT} / \mathrm{ETH}$ or PAS or $\mathrm{CS})$ & 57 & 367 & 16 & 5 & 41 & 12 & 35 & 179 & 20 & 15 & 74 & 20 & 112 & 661 & 17 \\
\hline $\mathrm{FQ}+(\mathrm{PT} / \mathrm{ETH}$ or PAS or $\mathrm{CS})+\mathrm{AMOX}$ & 1 & 367 & 0 & 3 & 41 & 7 & 8 & 179 & 4 & 4 & 74 & 5 & 16 & 661 & 2 \\
\hline Type 4: regimen includes INJ and FQ & 275 & 367 & 75 & 28 & 41 & 68 & 133 & 179 & 74 & 49 & 74 & 66 & 485 & 661 & 73 \\
\hline INJ+FQ only & 2 & 367 & 1 & 0 & 41 & 0 & 0 & 179 & 0 & 0 & 74 & 0 & 2 & 661 & 0 \\
\hline INJ+FQ+(PT/ETH or PAS or CS) & 252 & 367 & 69 & 25 & 41 & 61 & 81 & 179 & 45 & 29 & 74 & 39 & 387 & 661 & 59 \\
\hline INJ+FQ+(PT/ETH or PAS or CS)+AMOX & 21 & 367 & 6 & 3 & 41 & 7 & 52 & 179 & 29 & 20 & 74 & 27 & 96 & 661 & 15 \\
\hline
\end{tabular}

AMOX, amoxicillin/clavulanate; CS, cycloserine; FLD, first-line drug FQ, fluoroquinolones; INJ, second-line injectable agents; MDR, multidrug resistance, no additional resistance to FQs or second-line injectable agents; PAS, $p$-aminosalicylic acid; PT/ETH, prothionamide/ethionamide; SLD, second-line drugs; XDR, extensive drug resistance.

resistance do not necessarily impact on strain transmissibility. ${ }^{21}$ Indeed, presence of fitness-restoring mutations and those supporting drug resistance acquisition (ie, eis) have been identified in clades of Beijing family of strains ${ }^{22}$ that cause the majority of MDR-TB/XDR-TB in Eastern Europe. ${ }^{22}$

HIV infection played a crucial role in reducing chances to survive. Between $0 \%$ in Romania and $7 \%$ of patients in Estonia were coinfected with HIV; although routine HIV testing is not implemented everywhere and in particular is selectively offered in Lithuania.

The treatment was largely based on the administration of five drugs. Although these drugs being active based on the DST results, many patients still die too early for their age: half of the patients survived only 5.9 years after the diagnosis of MDR-TB/ XDR-TB, thus having only marginally better survival to the patients infected with drug-susceptible TB in the preantibiotic era. The median survival of those coinfected with HIV was just 1.9 years. From a public health point of view, it is of note that many of these patients with $\mathrm{TB}$ are infectious for a significant period of time and likely to contribute to the spread of MDR-TB/XDR-TB within the community and TB clinics. While in the majority of the patients culture conversion was achieved within the first 12 months of treatment; $13 \%$ of patients remained culture-positive throughout the entire treatment cycle and in $14 \%$ of patients a reversion was observed.

A recent meta-analysis by Falzon et $a l^{21}$ established that additional resistance to FQs (without as well as with additional resistance to injectables) has a negative impact on treatment outcomes of patients with MDR-TB. In our study, additional drug resistance in patients with MDR-TB including XDR had no significant effect on survival which is in line with the findings of other recent studies from Eastern Europe: Russia, ${ }^{23}$ Lithuania, ${ }^{14}$ Latvia $^{24}$ and might be explained by a very low proportion of patients with 'classic' MDR-TB without resistance to any other FLD or SLD. Most of the patients with MDR-TB would be 'near' XDR-TB effectively narrowing the difference between 'MDR' and 'XDR' survival. Increased virulence of the local strains and their ability to cause more severe disease irrespective of resistance pattern might be another reason. Besides, the effect of social factors such as high alcohol consumption, poor nutrition (which was not assessed within the scope of this work) might have overwhelming effects leading to premature death and mitigating the true effect of increasing drug resistance. Indeed, a recent study from Estonia confirmed that the excess 


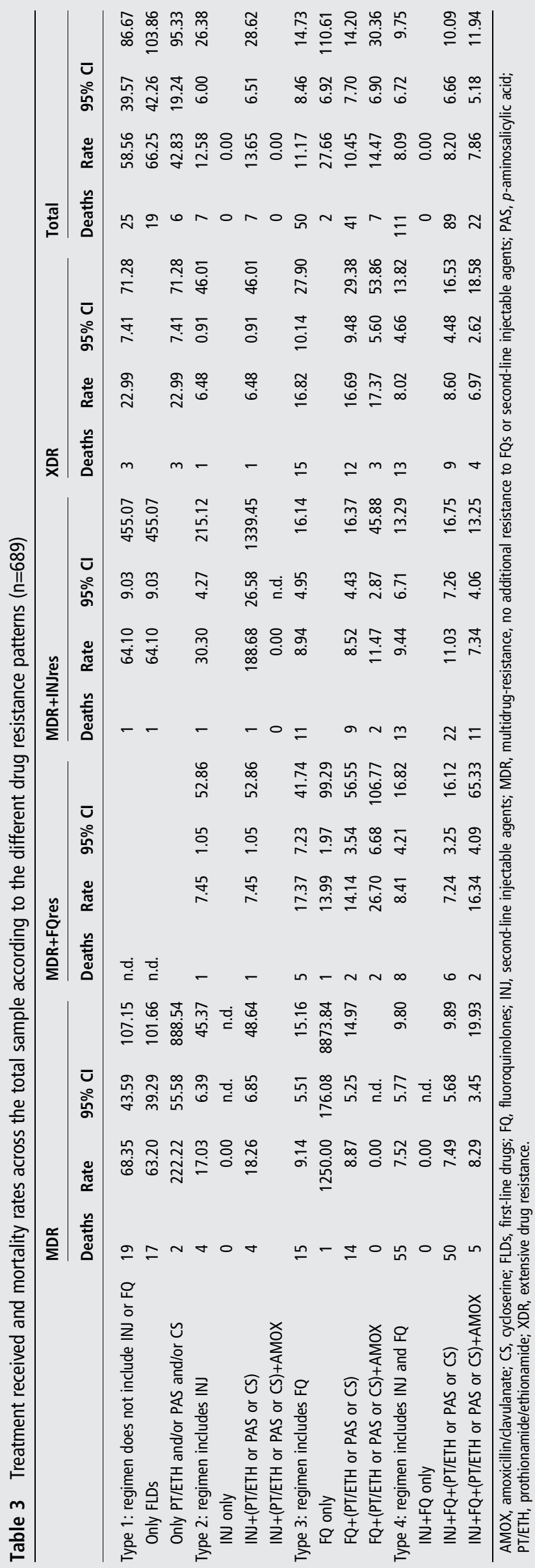

mortality due to other causes in patients with TB was largely alcohol-related and smoking-related. ${ }^{15}$ Despite the known negative impact of alcohol on TB treatment, that is, impaired immune response and increased risks of adverse effects, ${ }^{25}{ }^{26}$ the concurrent treatment of alcoholism as a medical problem is largely ignored by most of the TB control programmes in Eastern Europe. Two positive examples from Tomsk, Russia ${ }^{27}$ and Estonia ${ }^{28}$ have demonstrated significant improvement in treatment outcomes when using an integrated approach to management of patients with TB that addressed alcohol dependency treatment.

Our study demonstrated that mortality rates were highest when INJs and FQs were not part of the treatment irrespective of the resistance pattern; in all resistance groups including XDR, the regimen that included both INJs and FQs dramatically lowered mortality rates across the whole sample. We believe that these findings point towards a possible role of FQs and INJs in the treatment of MDR-TB cases with diagnosed in vitro resistance to these agents. In line with Dheda et $a l^{29}$ and Jacobson $\mathrm{K}$ et al ${ }^{16}$ our data suggest that inclusion of the latest generation FQs in XDR-TB treatment regimen in the absence of the specific results of DST to moxifloxacin is beneficial and needs to be considered.

The results showed that in patients without in vitro diagnosed resistance to ofloxacin administration of a later generation $\mathrm{FQ}$ (in this case, moxifloxacin) might be more advantageous compared with the use of older generation agents. However, in ofloxacin-resistant patients the choice of a FQ did not seem to make a difference. The beneficial effect of the FQs on survival in patients with ofloxacin-resistant XDR-TB was demonstrated previously in a South African cohort, ${ }^{29}$ in a Russian cohort ${ }^{23}$ as well as in a recent meta-analysis ${ }^{16}$ and might be explained by incomplete cross-resistance within the quinolone class ${ }^{30}$ and by the higher bactericidal activity of late generation FQs enabling them to overcome low-level resistance diagnosed in vitro. ${ }^{16}$ Introduction of routine in vitro DST to later generation FQs is very important and would better guide the therapy.

The study has several limitations. Its design did not allow the analysis of the length of treatment with each drug; besides the low case numbers per drug resistance pattern receiving particular treatment regimens permitted us to use the broader categories only. Information on antiretroviral therapy was available for a few patients infected with HIV only and therefore we could not assess its role on survival in patients with HIV coinfection. Reported alcohol and recreational drug abuse might be prone to a recall and reporting bias leading to potential underestimating of their roles in mortality.

In conclusion, the burden of MDR-TB and XDR-TB in Eastern European settings is high. While effective diagnostic and treatment strategies focus on prevention of drug-resistance development during treatment in initially susceptible cases, every effort should be made to stop further transmission of drug-resistant strains through improvements in infection control.

Improved drug regimens and early onset of treatment with at least four active drugs are absolutely essential in improving outcome. Additionally, to push the survival of the patients with MDR-TB and XDR-TB beyond that if no treatment was given; provision of social support to the patients with MDR-TB/ XDR-TB aiming to improve their adherence to treatment (eg, of incentives and enablers) must become an integral part of national TB control programmes. Correct identification of alcohol use disorders and their management are of vital importance for ensuring the success of treatment. ${ }^{19}{ }^{31}$ HIV testing 
Table 4 Factors associated with mortality across the total sample $(N=657$, deaths $N=182)$ accounting for interaction between drug resistance pattern and regimen type

\begin{tabular}{|c|c|c|c|c|c|c|c|c|c|}
\hline \multirow{2}{*}{$\begin{array}{l}\text { Factor } \\
\text { Age group (years) }\end{array}$} & \multirow[b]{2}{*}{$<30$} & \multirow{2}{*}{$\begin{array}{l}\text { Crude HR } \\
\text { Reference }\end{array}$} & \multicolumn{2}{|c|}{$95 \% \mathrm{Cl}$} & \multirow[t]{2}{*}{$\mathrm{p}$ Value } & \multirow{2}{*}{$\begin{array}{l}\text { Adjusted HR } \\
\text { Reference }\end{array}$} & \multicolumn{2}{|c|}{$95 \% \mathrm{Cl}$} & \multirow[t]{2}{*}{$\mathrm{p}$ Value } \\
\hline & & & & & & & & & \\
\hline & $30-39$ & 1.77 & 0.94 & 3.33 & 0.077 & 1.43 & 0.70 & 2.96 & 0.329 \\
\hline & $40-49$ & 2.62 & 1.44 & 4.77 & 0.002 & 2.22 & 1.13 & 4.34 & 0.020 \\
\hline & $50-59$ & 2.69 & 1.49 & 4.86 & 0.001 & 2.18 & 1.10 & 4.31 & 0.025 \\
\hline & $\geq 60$ & 4.20 & 2.26 & 7.83 & $<0.001$ & 2.47 & 1.18 & 5.17 & 0.017 \\
\hline Gender & $\begin{array}{l}\text { Male } \\
\text { Female }\end{array}$ & $\begin{array}{l}1.99 \\
\text { Reference }\end{array}$ & 1.37 & 2.90 & $<0.001$ & $\begin{array}{l}2.00 \\
\text { Reference }\end{array}$ & 1.27 & 3.14 & 0.003 \\
\hline Living settings & $\begin{array}{l}\text { Urban } \\
\text { Rural }\end{array}$ & $\begin{array}{l}0.92 \\
\text { Reference }\end{array}$ & 0.70 & 1.20 & 0.527 & & & & \\
\hline Employment & Unemployed & 2.52 & 1.62 & 3.90 & $<0.001$ & 1.48 & 0.90 & 2.41 & 0.120 \\
\hline & $\begin{array}{l}\text { Retired } \\
\text { Employed }\end{array}$ & $\begin{array}{l}3.67 \\
\text { Reference }\end{array}$ & 2.14 & 6.30 & $<0.001$ & $\begin{array}{l}2.69 \\
\text { Reference }\end{array}$ & 1.47 & 4.94 & 0.001 \\
\hline Smokingt & $\begin{array}{l}\text { Yes } \\
\text { No }\end{array}$ & $\begin{array}{l}1.65 \\
\text { Reference }\end{array}$ & 1.10 & 2.49 & 0.016 & & & & \\
\hline Alcohol abuse & $\begin{array}{l}\text { Yes } \\
\text { No }\end{array}$ & $\begin{array}{l}2.29 \\
\text { Reference }\end{array}$ & 1.68 & 3.12 & $<0.001$ & $\begin{array}{l}1.70 \\
\text { Reference }\end{array}$ & 1.16 & 2.47 & 0.006 \\
\hline Recreational drug use & $\begin{array}{l}\text { Yes } \\
\text { No }\end{array}$ & $\begin{array}{l}1.05 \\
\text { Reference }\end{array}$ & 0.57 & 1.93 & 0.871 & & & & \\
\hline HIV coinfection & Negative & Reference & & & & Reference & & & \\
\hline & Positive & 3.26 & 1.71 & 6.22 & $<0.001$ & 2.53 & 1.19 & 5.39 & 0.016 \\
\hline & Not tested & 1.29 & 0.97 & 1.71 & 0.080 & 0.68 & 0.47 & 1.01 & 0.056 \\
\hline Conditions other than HIV* & $\begin{array}{l}\text { Yes } \\
\text { No }\end{array}$ & $\begin{array}{l}2.63 \\
\text { Reference }\end{array}$ & 1.69 & 4.09 & $<0.001$ & $\begin{array}{l}2.33 \\
\text { Reference }\end{array}$ & 1.34 & 4.05 & 0.003 \\
\hline New or retreatment case & $\begin{array}{l}\text { Retreatment case } \\
\text { New case }\end{array}$ & $\begin{array}{l}0.90 \\
\text { Reference }\end{array}$ & 0.68 & 1.21 & 0.500 & & & & \\
\hline Pulmonary or extrapulmonary & $\begin{array}{l}\text { Both pulmonary and extrapulmonary } \\
\text { Pulmonary only }\end{array}$ & $\begin{array}{l}2.30 \\
\text { Reference }\end{array}$ & 1.36 & 3.89 & 0.002 & $\begin{array}{l}2.65 \\
\text { Reference }\end{array}$ & 1.45 & 4.83 & 0.002 \\
\hline Presence of cavities & $\begin{array}{l}\text { Yes } \\
\text { No }\end{array}$ & $\begin{array}{l}1.19 \\
\text { Reference }\end{array}$ & 0.91 & 1.57 & 0.206 & & & & \\
\hline Smear positivity at diagnosis & $\begin{array}{l}\text { Positive } \\
\text { Negative }\end{array}$ & $\begin{array}{l}1.48 \\
\text { Reference }\end{array}$ & 1.02 & 2.16 & 0.041 & $\begin{array}{l}1.24 \\
\text { Reference }\end{array}$ & 0.83 & 1.86 & 0.296 \\
\hline Treatment regimen $\ddagger$ & Resistance pattern§ & & & & & & & & \\
\hline \multirow[t]{4}{*}{ Includes INJ+FQ } & MDR & Reference & & & & Reference & & & \\
\hline & $\mathrm{MDR}+\mathrm{FQ}$ resistance & 1.05 & 0.50 & 2.22 & 0.892 & 1.10 & 0.51 & 2.35 & 0.809 \\
\hline & MDR+INJ resistance & 1.13 & 0.81 & 1.93 & 0.310 & 1.20 & 0.77 & 1.87 & 0.419 \\
\hline & $\mathrm{XDR}$ & 1.05 & 0.57 & 1.92 & 0.884 & 1.07 & 0.57 & 2.00 & 0.830 \\
\hline \multirow[t]{4}{*}{ Includes FQ, no INJ } & MDR & 1.18 & 0.67 & 2.09 & 0.570 & 1.11 & 0.60 & 2.05 & 0.747 \\
\hline & $\mathrm{MDR}+\mathrm{FQ}$ resistance & 2.16 & 0.86 & 5.42 & 0.102 & 1.07 & 0.35 & 3.31 & 0.900 \\
\hline & MDR+INJ resistance & 1.15 & 0.60 & 2.20 & 0.674 & 0.95 & 0.47 & 1.90 & 0.874 \\
\hline & XDR & 2.05 & 1.14 & 3.68 & 0.016 & 2.41 & 1.28 & 4.54 & 0.007 \\
\hline \multirow[t]{4}{*}{ Includes INJ, no FQ } & MDR & 2.16 & 0.78 & 6.00 & 0.138 & 3.39 & 1.17 & 9.81 & 0.024 \\
\hline & $\mathrm{MDR}+\mathrm{FQ}$ resistance & 1.10 & 0.14 & 7.33 & 0.990 & 0.73 & 0.10 & 5.48 & 0.762 \\
\hline & MDR+INJ resistance & 4.08 & 0.56 & 29.53 & 0.164 & 2.30 & 0.29 & 17.98 & 0.428 \\
\hline & XDR & 0.74 & 0.10 & 5.42 & 0.768 & 0.26 & 0.03 & 2.06 & 0.202 \\
\hline \multirow[t]{4}{*}{ No INJ, no FQ } & MDR & 8.23 & 4.84 & 14.01 & $<0.001$ & 8.74 & 4.84 & 15.77 & 0.000 \\
\hline & $\mathrm{MDR}+\mathrm{FQ}$ resistance & Empty & & & & & & & \\
\hline & MDR+INJ resistance & 1.13 & 0.81 & 1.93 & 0.310 & 1.20 & 0.77 & 1.87 & 0.419 \\
\hline & XDR & 2.82 & 0.87 & 9.07 & 0.083 & 3.78 & 1.13 & 12.65 & 0.031 \\
\hline
\end{tabular}

*Including hepatitis B, hepatitis C, chronic liver condition, other immunocompromised conditions (including autoimmune disorders) and cancer.

†Variable with a limited number of observations were not included into Cox multivariable regression model.

fInteraction term between treatment regimen and resistance pattern used in the unadjusted and the adjusted analyses.

INJ, second-line injectable drugs; MDR, multidrug resistance, no additional resistance to FQs or injectables; FQ, fluoroquinolones; XDR, extensive drug resistance.

should be expanded and antiretroviral therapy offered to every patient diagnosed as its early administration has been shown to substantially improve survival in patients with concomitant HIV/AIDS. ${ }^{29} 3233$ Similarly, more efforts should be focused on expansion of hepatitis $\mathrm{B}$ and $\mathrm{C}$ detection with appropriate treatment offered.

Prospective in vitro and clinical studies should provide evidence for the best use of FQs and INJs in treatment of patients with XDR-TB and pre-XDR-TB; meanwhile testing for later generation FQs could provide more exact information in settings with the high XDR-TB burden.

It is crucial to preserve susceptibility to the main SLDs in patients with MDR-TB and to achieve cure. We hope for shorter and more effective regimens containing new drugs to become available soon; however we need to simultaneously address other social, demographic and programmatic factors affecting treatment outcome and survival if these new regimens are to remain effective. 


\section{Author affiliations}

${ }^{1}$ Blizard Institute, Queen Mary, University of London, London, UK

2Department of Infectious Diseases, Imperial College London, London, UK

${ }^{3}$ Department for Infectious Disease Epidemiology, Robert Koch Institute, Berlin, Germany

${ }^{4}$ N.V. Postnikov Samara Region Clinical Tuberculosis Dispensary, Samara, Russia "Department of Mycobacteriology, State Agency "Infectology Center of Latvia", Clinic for Tuberculosis and Lung Diseases, "Upeslejas" Stopinunovads, Riga, Latvia ${ }^{6}$ United Laboratory, Department of Mycobacteriology, Tartu University Hospital, Tartu, Estonia

${ }^{7}$ National Tuberculosis and Infectious Diseases University Hospital, Vilnius, Lithuania

${ }^{8}$ Marius Nasta Institute of Pneumology, Bucharest, Romania

Acknowledgements We would like to thank Ekaterina Koshkarova for support with the data management.

Contributors $Y B, L F, O$ and FD drafted the manuscript; all authors reviewed and edited the manuscript. All authors participated in the study design. YB, OI, VN and FD supervised the project. BR, MD, KJ, ED, BR and CMP conducted the study. LF and $\mathrm{Ol}$ conducted the statistical analysis.

Funding The research leading to these results has received funding from the European Community's Seventh Framework Programme (FP7/2007-2013) under grant agreement FP7-223681.

Competing interests None declared.

Ethics approval The project was reviewed and approved by the Ethics Review Committees of the University of Tartu and Riga Stradins University and received a waiver of informed consent as anonymised data were used. Local Ethics Committees exempted the study from an ethics review in Lithuania and Romania. The study was approved by Queen Mary College Research Ethics Committee.

Provenance and peer review Not commissioned; externally peer reviewed.

\section{REFERENCES}

1 Acosta CD, Dadu A, Ramsay A, et al. Drug-resistant tuberculosis in Eastern Europe: challenges and ways forward. Public Health Action 2014;4:3-12.

2 World Health Organisation. Global Tuberculosis Report. Geneva: WHO/HTM/TB/ 2015.22, 2015.

3 Lucenko I, Riekstina V, Perevoscikovs J, et al. Treatment outcomes among drug-susceptible tuberculosis patients in Latvia, 2006-2010. Public Health Action 2014:4:54-8.

4 Dye C. Doomsday postponed? Preventing and reversing epidemics of drug-resistant tuberculosis. Nat Rev Microbiol 2009;7:81-7.

5 Leimane V, Dravniece G, Riekstina V, et al. Treatment outcome of multidrug/ extensively drug-resistant tuberculosis in Latvia, 2000-2004. Eur Respir J 2010;36:584-93.

6 Skripconoka V, Danilovits M, Pehme L, et al. Delamanid improves outcomes and reduces mortality in multidrug-resistant tuberculosis. Eur Respir J 2013;41:1393-400.

7 Didilescu C, Popescu G, Cioran N, et al. [Mortality of tuberculosis in Romania, a marker for severity of the endemic]. Pneumologia 2012;61:150-2.

8 Zignol M, Dara M, Dean AS, et al. Drug-resistant tuberculosis in the WHO European Region: an analysis of surveillance data. Drug Resist Updat 2013;16:108-15.

9 Merker M, Blin C, Mona S, et al. Evolutionary history and global spread of the Mycobacterium tuberculosis Beijing lineage. Nat Genet 2015;47:242-9.

10 Mokrousov I. Insights into the origin, emergence, and current spread of a successful Russian clone of Mycobacterium tuberculosis. Clin Microbiol Rev 2013;26:342-60.

11 Joint United Nations Programme on HIVIAIDS (UNAIDS). Global Report: UNAIDS report on the global AIDS epidemic 2013 2UNAIDS/JC2502/1/E. Geneva, 2013.
12 Falzon D, Mirzayev F, Wares F, et al. Multidrug-resistant tuberculosis around the world: what progress has been made? Eur Respir J 2015;45:150-60.

13 Gandhi NR, Nunn P, Dheda K, et al. Multidrug-resistant and extensively drug-resistant tuberculosis: a threat to global control of tuberculosis. Lancet 2010;375:1830-43.

14 Balabanova Y, Radiulyte B, Davidaviciene E, et al. Survival of drug resistant tuberculosis patients in Lithuania: retrospective national cohort study. BMJ Open 2011:1:e000351.

15 Blöndal K, Rahu K, Altraja A, et al. Overall and cause-specific mortality among patients with tuberculosis and multidrug-resistant tuberculosis. Int I Tuberc Lung Dis 2013;17:961-8

16 Jacobson KR, Tierney DB, Jeon $C Y$, et al. Treatment outcomes among patients with extensively drug-resistant tuberculosis: systematic review and meta-analysis. Clin Infect Dis 2010;51:6-14.

17 Keshavjee S, Gelmanova IY, Pasechnikov AD, et al. Treating multidrug-resistant tuberculosis in Tomsk, Russia: developing programs that address the linkage between poverty and disease. Ann N Y Acad Sci 2008:1136:1-11.

18 Lefebvre N, Falzon D. Risk factors for death among tuberculosis cases: analysis of European surveillance data. Eur Respir J 2008:31:1256-60.

19 Mathew TA, Yanov SA, Mazitov R, et al. Integration of alcohol use disorders identification and management in the tuberculosis programme in Tomsk Oblast, Russia. Eur J Public Health 2009;19:16-18.

20 Siddiqi S, Ahmed A, Asif S, et al. Direct drug susceptibility testing of Mycobacterium tuberculosis for rapid detection of multidrug resistance using the Bactec MGIT 960 system: a multicenter study. J Clin Microbiol 2012;50:435-40.

21 Falzon D, Gandhi N, Migliori GB, et al. Resistance to fluoroquinolones and second-line injectable drugs: impact on multidrug-resistant TB outcomes. Eur Respir J 2013:42:156-68.

22 Casali N, Nikolayevskyy V, Balabanova Y, et al. Evolution and transmission of drug-resistant tuberculosis in a Russian population. Nat Genet 2014;46:279-86.

23 Keshavjee S, Gelmanova IY, Farmer PE, et al. Treatment of extensively drug-resistant tuberculosis in Tomsk, Russia: a retrospective cohort study. Lancet 2008:372:1403-9.

24 Kuksa L, Riekstina V, Leimane V, et al. Multi- and extensively drug-resistant tuberculosis in Latvia: trends, characteristics and treatment outcomes. Public Health Action 2014;4(Suppl 2):47-53.

25 Duraisamy K, Mrithyunjayan S, Ghosh S, et al. Does Alcohol consumption during multidrug-resistant tuberculosis treatment affect outcome?. A population-based study in Kerala, India. Ann Am Thorac Soc 2014:11:712-18.

26 Liang Y, Harris FL, Brown LA. Alcohol induced mitochondrial oxidative stress and alveolar macrophage dysfunction. Biomed Res Int 2014;2014:371593.

27 Shin S, Livchits V, Connery HS, et al. Effectiveness of alcohol treatment interventions integrated into routine tuberculosis care in Tomsk, Russia. Addiction 2013;108:1387-96.

28 World Health Organisation. Collaborative action on tuberculosis and alcohol abuse in Estonia: First report of a demonstration project. Copenhagen: WHO, 2013.

29 Dheda K, Shean K, Zumla A, et al. Early treatment outcomes and HIV status of patients with extensively drug-resistant tuberculosis in South Africa: a retrospective cohort study. Lancet 2010;375:1798-807.

30 Kam KM, Yip CW, Cheung TL, et al. Stepwise decrease in moxifloxacin susceptibility amongst clinical isolates of multidrug-resistant Mycobacterium tuberculosis: correlation with ofloxacin susceptibility. Microb Drug Resist 2006;12:7-11.

31 Miller AC, Gelmanova IY, Keshavjee $S$, et al. Alcohol use and the management of multidrug-resistant tuberculosis in Tomsk, Russian Federation. Int I Tuberc Lung Dis 2012:16:891-6

32 Glaziou P, Sismanidis C, Floyd K, et al. Global Epidemiology of Tuberculosis. Cold Spring Harb Perspect Med 2014;5:a017798.

33 Pietersen E, Ignatius E, Streicher EM, et al. Long-term outcomes of patients with extensively drug-resistant tuberculosis in South Africa: a cohort study. Lancet 2014;383:1230-9. 